\title{
Sound velocity of hcp-Fe at high pressure: experimental constraints, extrapolations and comparison with seismic models
}

\author{
Daniele Antonangeli ${ }^{*}$ and Eiji Ohtani ${ }^{2}$
}

\begin{abstract}
Determining the sound velocity of iron under extreme thermodynamic conditions is essential for a proper interpretation of seismic observations of the Earth's core but is experimentally challenging. Here, we review techniques and methodologies used to measure sound velocities in metals at megabar pressures, with specific focus on the compressional sound velocity of hexagonal close-packed iron. A critical comparison of literature results, coherently analyzed using consistent metrology (pressure scale, equation of state), allows us to propose reference relations for the pressure and density dependence of the compressional velocity of hexagonal close-packed iron at ambient temperature. This provides a key base line upon which to add complexity, including high-temperature effects, pre-melting effects, effects of nickel and/or light element incorporation, necessary for an accurate comparison with seismic models, and ultimately to constrain Earth's inner core composition.
\end{abstract}

Keywords: Sound velocity measurements; High pressure; hcp-Fe; Extrapolation schemes; Earth's inner core; Comparison with seismic models

\section{Review}

Iron $(\mathrm{Fe})$ has always attracted considerable interest in fundamental physics and chemistry, as well as materials science, due to its primary role in a great variety of technological applications. The physical properties of Fe at high pressure and high temperature also have unique relevance for Earth and planetary science, since all the telluric planets share the same basic layered nature: a central metallic core, made mostly of iron, surrounded by a silicate mantle and a thin, chemically differentiated crust. Thus, the experimental characterization and theoretical calculations of the physical properties of iron at relevant thermodynamic conditions are crucial in refining the chemical composition and dynamics of the Earth's core and of telluric planetary cores more generally.

Direct sampling of the Earth's deepest regions, including the core, is impossible. Amongst the remote sensing techniques we have to rely on, seismology is certainly

\footnotetext{
* Correspondence: daniele.antonangeli@impmc.upmc.fr

'Institut de Minéralogie, de Physique des Matériaux, et de Cosmochimie (IMPMC), UMR CNRS 7590, Sorbonne Universités - UPMC Univ. Paris 6, Muséum National d'Histoire Naturelle, IRD unité 206, 75252 Paris, France Full list of author information is available at the end of the article
}

one of the most direct. Conventional, radially averaged, one-dimensional seismic models, such as the Preliminary Reference Earth Model (PREM, Dziewonski and Anderson, 1981), provide a generalized description of the evolution of the sound velocities $\left(V_{\mathrm{P}}\right.$ and $\left.V_{\mathrm{S}}\right)$ and density $(\rho)$ with depth. However, to derive an accurate compositional model, these seismic observations have to be combined with experiments or calculations constraining the density and elastic properties of highly compressed candidate materials. In this respect, compressional-wave $\left(V_{\mathrm{P}}\right)$ and shear-wave $\left(V_{\mathrm{S}}\right)$ sound velocities play a fundamental role, as two of the few parameters that can be directly compared with the seismic observations.

In the early 1950s, Birch used shock wave measurements to support the notion that the Earth's core was mainly composed of iron (Birch, 1952). Since then, a large number of experimental and theoretical studies have addressed the sound velocities of solid iron at extreme conditions. Over the last 15 years, a great deal of effort has been devoted to the development of experiments capable of probing elasticity and sound velocity of opaque metallic samples at high static pressure, with iron providing much of the motivation. Current data 
sets, however, even including those based on indirect determinations, are generally limited to pressures of the order of 100 to $200 \mathrm{GPa}$ at ambient temperature, and very few results exist at simultaneous high pressure and temperature. Most importantly, different studies are not always in agreement. Thus, even neglecting eventual corrections at high temperature, the extrapolations of results to the pressures of the Earth's inner core (330 $\mathrm{GPa}<P<360 \mathrm{GPa})$ come with large uncertainties. These limitations become very critical when attempting to estimate the nature and amounts of light elements, whose presence as impurities in the inner core is required to modify the density and velocity of iron to match seismic observations. For instance, models based on linear extrapolations of $V_{\mathrm{P}}-\rho$ relation argue for about $2 \mathrm{wt} \%$ silicon alloyed with iron in the inner core (Badro et al., 2007; Antonangeli et al., 2010), while a recent model using a power law for the $V_{\mathrm{P}}-\rho$ extrapolation leads to 8 wt\% silicon (Mao et al., 2012).

In this paper, we intend to briefly review the techniques for sound velocity determinations. Literature data will be examined and compared to ultimately propose a reference for the pressure and density evolution of compressional sound velocity of hexagonal closed-packed (hcp) iron, the polymorph of iron observed to be stable at Earth's inner core conditions (Tateno et al., 2010). Finally, the proposed velocity-density relationship will be discussed in relation to potential high-temperature effects.

\section{Experimental techniques for sound velocity determination under static high pressure}

In the 1950s, shock wave measurements provided the bulk sound velocity $\left(V_{\phi}\right)$ of iron along the Hugoniot curve at multi-megabar pressure (Birch, 1952), and the compressional sound velocity of solid iron has been determined along the Hugoniot up to melting (approximately 205 GPa) (Brown and McQueen, 1986). Conversely, sound velocity measurements of iron under static compression turned out to be an experimental challenge.

Conventional pulse-echo ultrasonic techniques require sample dimensions large enough to avoid overlap of successive acoustic echoes and, hence, experimental volumes of several cubic millimeters, hardly compatible with experimentation at megabar pressures. As a result, the ultrasonic $V_{\mathrm{P}}$ and $V_{\mathrm{S}}$ have been reported for hcp-Fe at only 16.5 GPa (Mao et al., 1998). Gigahertz interferometry has been proposed as a possible solution to overcome these hindrances, but applications at very high pressure are still limited (e.g. Jacobsen et al., 2004). Only very recently, measurements of acoustic echoes in samples compressed in diamond anvil cells became possible thanks to the developments in time-resolved pump-probe techniques (laser ultrasonics), both in the nanosecond time scale (Chigarev et al., 2008) and in the picosecond time scale (Decremps et al., 2008). Measurements on iron provided $V_{\mathrm{P}}$ and $V_{\mathrm{S}}$ up to $23 \mathrm{GPa}$ (Chigarev et al., 2008) and of the only $V_{\mathrm{P}}$ up to $152 \mathrm{GPa}$ (Decremps et al., 2014).

Another classical method to obtain elasticity and sound velocity of samples at high pressure is Brillouin spectroscopy (e.g. Murakami et al., 2012), for which application is limited to optically transparent specimens. Impulsive stimulated light scattering (ISLS) has been developed to enable measurements on metallic samples but is sensitive to surface properties, and data analysis is relatively complex (e.g. Crowhurst et al., 2006). Nonetheless, aggregate sound velocities of hcp-Fe have been determined up to 115 GPa with this method (Crowhurst et al., 2004).

It is only with the advent of third-generation synchrotron sources and the development of X-ray diffraction and scattering techniques in conjunction with the use of diamond anvil cells that systematic experiments on iron and iron alloys at megabar pressure have become possible. The first attempts to obtain information on elasticity and sound velocity of hcp-Fe were based on X-ray diffraction (XRD) techniques. Lattice strain relations obtained by radial diffraction measurements on non-hydrostatically compressed iron were analyzed to estimate the singlecrystal elastic moduli, and hence the aggregate velocities, up to $220 \mathrm{GPa}$ at ambient temperature (Mao et al., 1998). Alternatively, Rietveld refinement has been exploited to estimate the Debye-Waller factor and then to calculate the aggregate velocities starting from X-ray diffraction measurements on hcp-Fe up to $300 \mathrm{GPa}$ and $1,200 \mathrm{~K}$ (Dubrovinski et al., 2001). Although such studies provide very valuable information, they only probe the sound velocities indirectly, relying heavily on interpretative schemes and assumptions in data analysis. Specifically, stress inhomogeneities resulting from grain-to-grain interaction in combination with effects of plastic deformation undermine the validity of the continuum elasticity theory used by Mao et al. (1998) to invert the singlecrystalline elastic moduli from the measured lattice strain (Merkel et al., 2006; 2009), while the results of Dubrovinski et al. (2001) explicitly depend on the assumed relation between the Debye-Waller temperature factor and the aggregate elastic properties.

In the early 2000s, two phonon-probe techniques were developed, almost in parallel: nuclear resonant inelastic $\mathrm{X}$-ray scattering (NRIXS or NIS) and inelastic X-ray scattering (IXS or HERIXS). NRIXS probes the partial projected phonon density of states of materials containing Mössbauer resonant isotopes. In the case of pure ${ }^{57} \mathrm{Fe}$, the total phonon density of states is probed. Amongst the various thermoelastic and vibrational parameters that can be derived, the Debye velocity can be obtained by a parabolic fit of the low-energy portion of the phonon density of states (Sturhahn and Jackson, 2007). Combining the measured Debye velocity with results from an equation of 
state (density and adiabatic bulk modulus), $V_{\mathrm{P}}$ and $V_{\mathrm{S}}$ can be determined. IXS, on the other hand, can be applied to any material and probes the momentum-resolved phonon dispersions. In the case of experiments on single crystals, the longitudinal and transverse sound velocities can be measured along any arbitrary direction, and all the independent elements of the elastic tensor can be obtained (e.g. Antonangeli et al., 2004a). $V_{\mathrm{P}}$ and $V_{\mathrm{S}}$ can then be derived by straightforward averaging. However, in the case of measurements on polycrystalline samples (as typically the case for experiments at very high pressures), the loss of directional information limits the measurements to the averaged phonon dispersions of the aggregate (Bosak et al., 2007). $V_{\mathrm{P}}$ can be then obtained from a sine fit to the aggregate phonon dispersion and $V_{\mathrm{S}}$ derived by combining the measured $V_{\mathrm{P}}$ with results from an equation of state (density and adiabatic bulk modulus) (Antonangeli et al., 2004b), but data analysis and interpretation now depend on how well the data sample the linear part of the phonon dispersion and on the knowledge of sample texture (Bosak et al., 2007).

Pioneering studies by IXS (Fiquet et al., 2001) and NRIXS (Mao et al., 2001) on hcp-Fe are contradictory. The linear extrapolation of the $V_{\mathrm{P}}-\rho$ relation proposed by IXS experiments up to $110 \mathrm{GPa}$ (Fiquet et al., 2001) is in close agreement with shock wave determinations (Brown and McQueen, 1986), suggesting that anharmonic effects at high temperature are negligible (Birch's law) and arguing for hcp-Fe slower than PREM at core densities. On the contrary, $V_{\mathrm{P}}$ derived from the phonon density of states measured by NRIXS up to 153 GPa (Mao et al., 2001) plots higher than shock wave determination (Brown and McQueen, 1986), indicating possible temperature effects, and extrapolates at core pressures to $V_{\mathrm{P}}$ higher than PREM. Later NRIXS and IXS studies, with progressively improved beamline performance (flux, focusing optics), improved statistics and improved sample environment (hydrostaticity and/or texture characterization), focused on anisotropy (Antonangeli et al., 2004b; Lin et al., 2010), high-temperature effects (Lin et al., 2005; Kantor et al., 2007; Antonangeli et al., 2008; Antonangeli et al., 2012; Ohtani et al., 2013), or effect of nickel and/or light element inclusion (Lin et al., 2003; Badro et al., 2007; Kantor et al, 2007; Gao et al., 2008; Fiquet et al., 2009; Antonangeli et al., 2010; Shibazaki et al., 2012; Kamada et al., 2014). In view of the specific characteristics of the two techniques and of the respective data analysis, today's general consensus is that, for measurements on polycrystalline samples, IXS provides more reliable $V_{\mathrm{P}}$ and NRIXS more reliable $V_{\mathrm{S}}$ (the Debye velocity measured directly by NRIXS is more heavily weighted in the determination of $V_{\mathrm{S}}$, while $V_{\mathrm{P}}$ determination is more dependent on the used equation of state; in comparison, IXS provides a direct determination of $V_{\mathrm{P}}$ while $V_{\mathrm{S}}$ depends on the choice of equation of state).

\section{Density dependence of the compressional sound velocity in hcp-Fe}

All the techniques discussed above have advantages and drawbacks. A critical discussion of each individual technique is beyond the scope of the present paper. In the following, we will only compare results obtained for polycrystalline hcp-Fe under static compression by different methods, with specific interest on the pressure and density dependence of the compressional sound velocity. Our choice in limiting detailed discussion to $V_{\mathrm{P}}$ is mainly driven by the fact that comparison with seismic models is more straightforward for the case of $V_{\mathrm{P}}$ than $V_{\mathrm{S}}$. This is because anharmonic and/or pre-melting effects at high temperature (e.g. Laio et al., 2000; Vočadlo et al., 2009; Sha and Cohen, 2010; Martorell et al., 2013), as well as the possible presence of fluid inclusion in the core (Vočadlo, 2007), or frequency-dependent viscoelastic relaxations (Jackson et al., 2000), are all expected to more strongly influence $V_{\mathrm{S}}$ than $V_{\mathrm{P}}$.

Comparison of results obtained by the various techniques, and sometime even the comparison of results obtained by the same technique, is complicated by dissimilar experimental strategies, differences amongst sample materials (isotopic compositions, degree of hydrostaticity, powders vs. foils, textures), and use of different pressure scales or equations of state (EOS). All these aspects have to be borne in mind when confronting different data sets. To allow for the most direct comparison, following Sakai et al. (2011; 2014), we rescaled reported pressures to the Pt scale of Matsui et al. (2009), which yields results quite consistent with the pressure scale of Dewaele et al. (2004) and Fei et al. (2007). When not otherwise specified, we use a thirdorder Birch-Murnagan relation with $V_{0}=22.468 \AA^{3} /$ unit cell (Dewaele et al. 2006), $K_{0}=155 \mathrm{GPa}$ and $K^{\prime}=5.37$ (Sakai et al., 2014) as the reference hcp-Fe EOS. To account for temperature effects, at least within the limit of a quasi-harmonic approximation (Antonangeli et al., 2008), we opted for the so-called Birch's diagram, a $V_{\mathrm{P}}-\rho$ representation of the data.

Taking a conservative approach, the ultrasonic determination of velocities for hcp-Fe reported in Mao et al. (1998) can be considered as an anchor point, yielding $V_{\mathrm{P}}=6,950 \pm 140 \mathrm{~m} / \mathrm{s}$ at $16.5 \mathrm{GPa}\left(\rho=8,981 \mathrm{~kg} / \mathrm{m}^{3}\right)$. Estimations of $V_{\mathrm{P}}$ starting from XRD measurements are one of the most indirect and model-dependent approach, but with the advantage of providing the largest coverage in pressure (and hence density) range (up to approximately $200 \mathrm{GPa}$ in Mao et al. (1998) and up to approximately $275 \mathrm{GPa}$ in Dubrovinski et al. (2001), once rescaling reported values to the Matsui $\mathrm{P}$ scale). $V_{\mathrm{P}}$ obtained as a function of density is illustrated in Figure 1. Besides the 


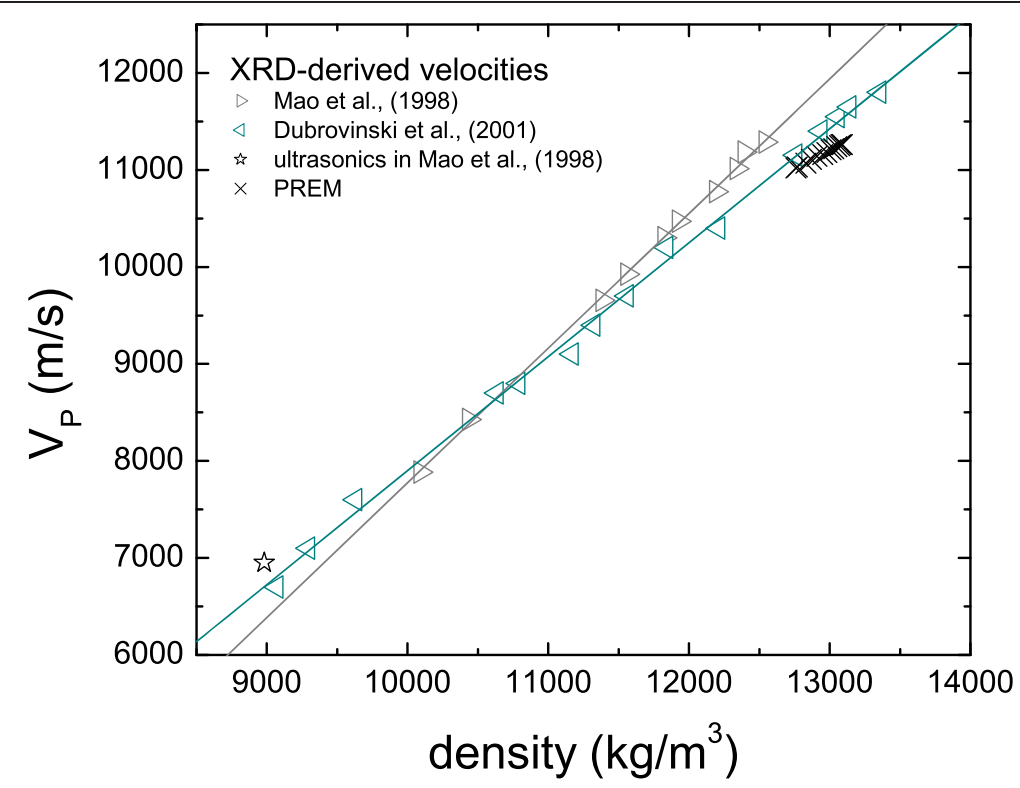

Figure 1 XRD-derived compressional sound velocity as a function of density. Grey right-pointing triangles (Mao et al., 1998); dark cyan left-pointing triangles (Dubrovinski et al., 2001). Lines are linear fit to the distinct experimental data sets. The star is the ultrasonic determination reported in Mao et al. (1998). PREM is shown as crosses.

absolute values for the velocities, it is very interesting to note that both data sets follow a linear density evolution up to densities exceeding those of the Earth's inner core. In both cases, hcp-Fe is suggested to have $V_{\mathrm{P}}$ higher than PREM at inner core density (Figure 1).

IXS experiments on hcp-Fe have been conducted over the last 15 years at the European Synchrotron Radiation Facility (ESRF) (Fiquet et al., 2001; Antonangeli et al., 2004b; Antonangeli et al., 2012) and more recently at the Advanced Photon Source (APS) (Mao et al., 2012) and at Spring-8 (Ohtani et al., 2013). This gives us the opportunity to compare measurements performed over time with the same instrument with similar experimental strategies, as well as results obtained by the same technique at different synchrotrons, often involving dissimilar experimental strategies. Importantly, in all cases, diffraction patterns have been collected in situ to obtain directly the densities, and the measured velocities do not depend upon any assumption concerning the equation of state. These aspects greatly facilitate direct comparison and allow evaluation of the finer aspects of the measurements such as instrument operation, energy and momentum resolution, analyzer settings, and sample preferred orientation. For a direct comparison, we only considered the ambient temperature measurements, even for the more recent studies including data at simultaneous highpressure and high-temperature conditions (Antonangeli et al., 2012; Mao et al., 2012; Ohtani et al., 2013).

The $V_{\mathrm{P}}$ results obtained at $300 \mathrm{~K}$ by Fiquet et al. (2001), Antonangeli et al. (2004b) and Antonangeli et al. (2012) using IXS are consistent but for the highest density point in Fiquet et al. (2001) (see Figure 2). In this pioneering IXS work (Fiquet et al., 2001), the phonon dispersion curve at $110 \mathrm{GPa}$ was constrained with only 2 points and the resulting $V_{\mathrm{P}}$ is not reliable enough. This low $V_{\mathrm{P}}$ value at $110 \mathrm{GPa}$ largely affected the extrapolation of the measured velocities to inner core densities, leading to extrapolated $V_{\mathrm{P}}$ well below PREM (Figure 2). Subsequent measurements (Antonangeli et al., 2004b; Antonangeli et al., 2012) have been carried out with improved instrument performances (increased flux, better focusing optics), constraining the phonon dispersion curves with more points (8 to 9 ) and with improved statistics. As a consequence, $V_{\mathrm{P}}$ was determined with progressively increased precision (error bars are not shown in Figure 2 for clarity) and the data obtained display less dispersion with respect to the $V_{\mathrm{P}}-\rho$ trend that extrapolates close to, but is slightly higher than, PREM (Figure 3). We note, however, that, as illustrated in Figure 2, the linear fits to the individual data sets are not strictly the same.

The $V_{\mathrm{P}}-\rho$ relation obtained from a linear fit to the combined data set of Antonangeli et al. (2004b) and Antonangeli et al. (2012) is compared to IXS results by Mao et al., (2013) and Ohtani et al. (2013) in Figure 3. While results by Antonangeli et al. (2004b; 2102) and by Ohtani et al. (2013) support a linear evolution of $V_{\mathrm{P}}$ with density (the two trends are almost parallel, only shifted in the absolute values by 200 to $300 \mathrm{~m} / \mathrm{s}$ ), Mao et al. (2012) proposed a sub-linear evolution, described by a power-law function. This sub-linear relation is also at odds with the $V_{\mathrm{P}}-\rho$ trend derived by the analysis of XRD 


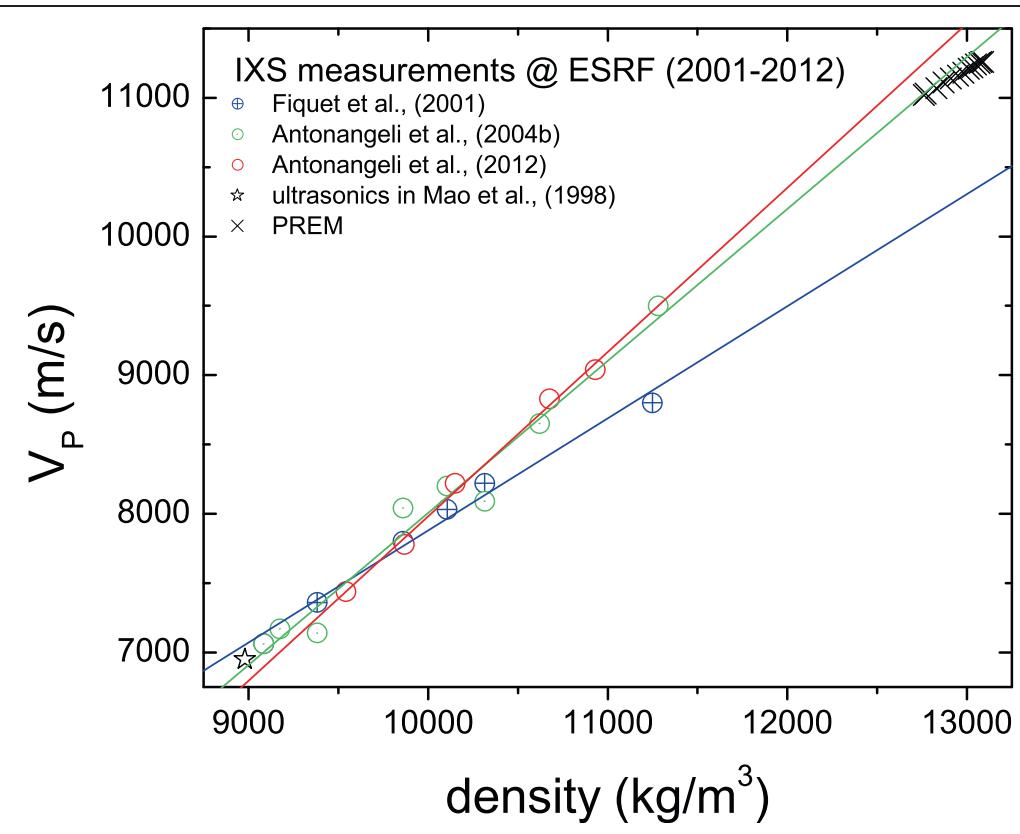

Figure 2 IXS compressional sound velocity as a function of density. Blue crossed circles (Fiquet et al., 2001); green dot centre circles (Antonangeli et al., 2004b); red open circles (Antonangeli et al., 2012). Lines are linear fits to the distinct experimental data sets. The star is the ultrasonic determination reported in Mao et al. (1998). PREM is shown as crosses.

measurements (Figure 1), which, as mentioned previously, covered the largest density range amongst all determinations. Similarly, it is not supported by results obtained by NRIXS or light scattering (Figures 4 and 5). As a direct consequence of the linear vs. power-law fit, $V_{\mathrm{P}}$ values of Ohtani et al. (2013) extrapolate to values higher than
PREM, and $V_{\mathrm{P}}$ by Antonangeli et al. (2004b; 2012) extrapolates close to, but slightly higher than, PREM, while $V_{\mathrm{P}}$ by Mao et al. (2012) extrapolates to values below PREM (Figure 3). Reasons for these differences can be at least qualitatively understood by considering similarities and distinctions in spectrometer operations. Both IXS

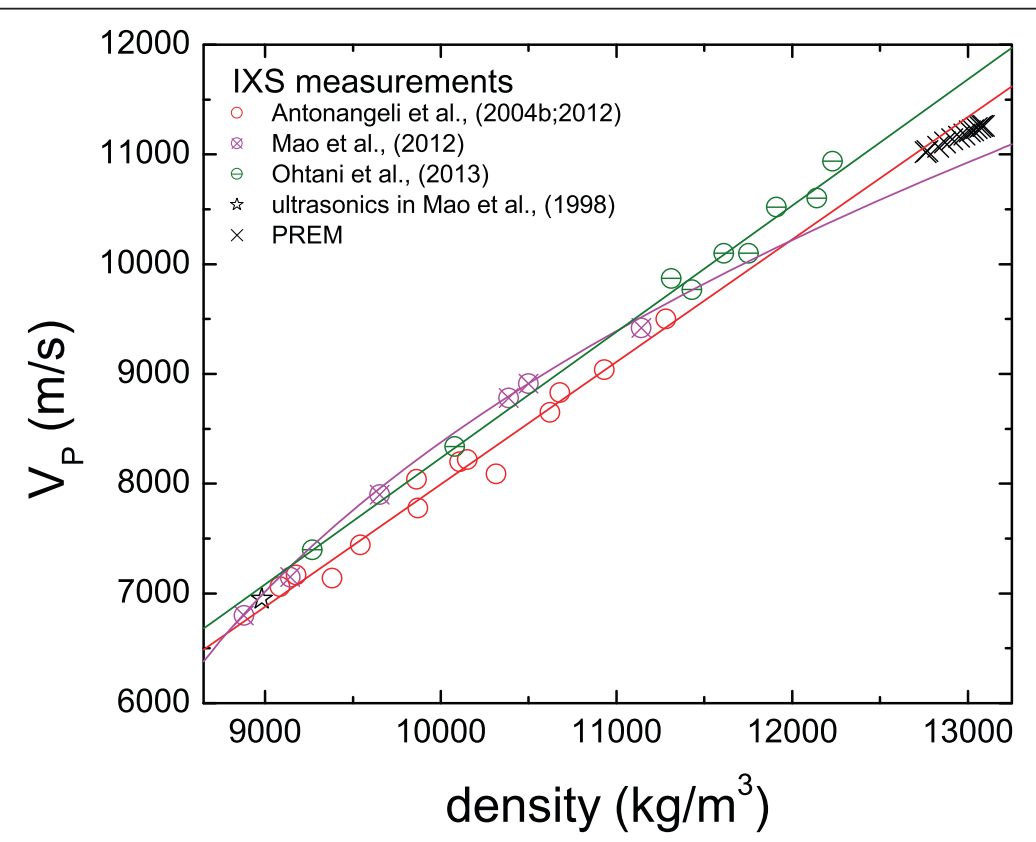

Figure 3 IXS compressional sound velocity as a function of density. Red open circles (Antonangeli et al., 2004b; 2012); magenta crossed circles (Mao et al., 2012); green lined circles (Ohtani et al., 2013). The curve and the lines are fit to the distinct experimental data sets. The star is the ultrasonic determination reported in Mao et al. (1998). PREM is shown as crosses. 


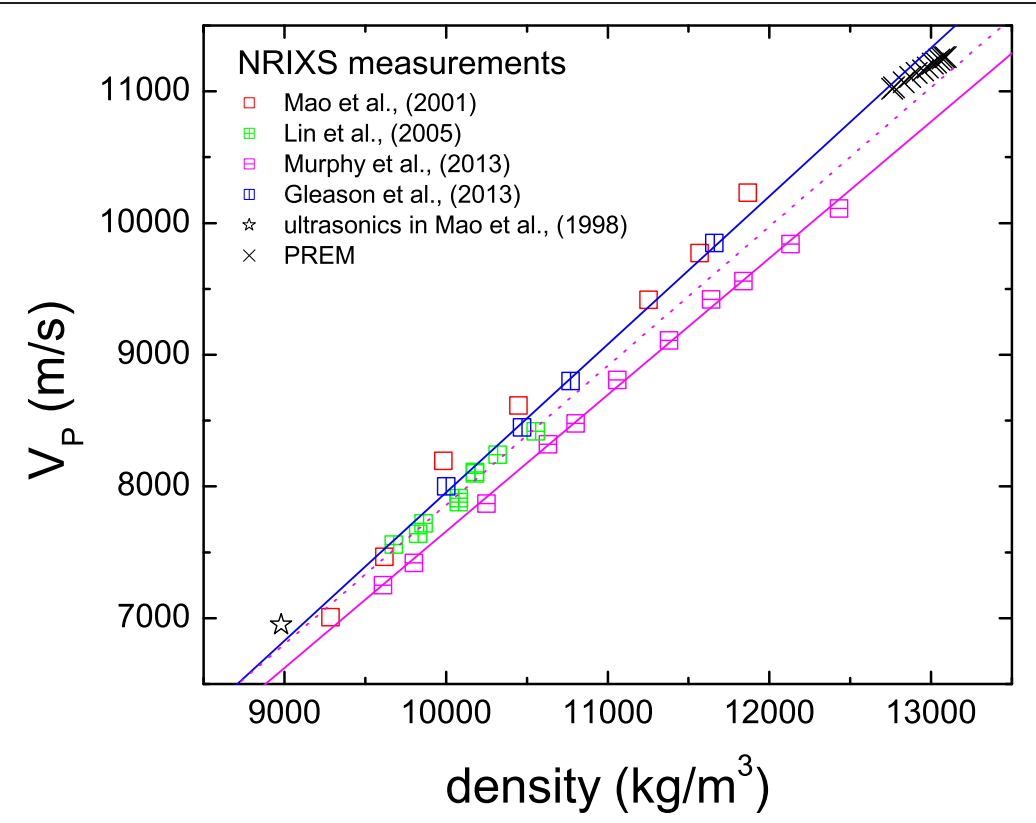

Figure 4 NRIXS compressional sound velocity as a function of density. Red squares (Mao et al., 2001); green crossed open squares (Lin et al., 2005); magenta horizontal-lined squares (Murphy et al., 2013); blue vertical-lined squares (Gleason et al., 2013). Solid lines are linear fits to the experimental data sets by Murphy et al. (2013) and Gleason et al. (2013). For comparison, the results by Murphy et al. (2013) with densities rescaled to those for iron of natural isotopic abundance (55.85 vs. $56.95 \mathrm{~g} / \mathrm{mol}$ ) are plotted as a dotted line. The star is the ultrasonic determination reported in Mao et al. (1998). PREM is shown as crosses.

spectrometers at ESRF and Spring-8 operate in backscattering geometry and scan energies by varying the temperature of the main monochromator, while the spectrometer at APS exploits a six-reflection inline monochromator. These technical aspects directly influence the temporal stability of the elastic energy and the data collection strategy (longer integration scans vs. sum of short scans). Another significant difference is in the number, relative position and momentum resolution of the points used to constrain the phonon dispersion within the first Brillouin zone: Antonangeli et al. (2012) used 8 to 9 points in the 3.5 to $12.5 \mathrm{~nm}^{-1}$ range, with a momentum resolution of $0.25 \mathrm{~nm}^{-1}$, Ohtani et al. (2013) used 4 points in the 8 to $12 \mathrm{~nm}^{-1}$ range, with a momentum resolution of $0.4 \mathrm{~nm}^{-1}$, and Mao et al. (2012) used 5 points in the 4 to $14 \mathrm{~nm}^{-1}$ range, with a momentum resolution of $0.7 \mathrm{~nm}^{-1}$. A detailed discussion of the influence of q-settings and qresolution on the derived velocity can be found in Bosak et al. (2007). Finally, especially when comparing the results of Antonangeli et al. (2012) with those of Ohtani et al. (2013), we cannot rule out a difference due to absolute energy calibration. Another non-negligible aspect to be considered in the comparison of various results is the nature of the specimens. Powder samples (Antonangeli et al., 2004b; Antonangeli et al., 2012; Ohtani et al. 2013) are more suitable than foils (Mao et al., 2012), which may have significant texture (rolling texture), that undermines the assumption of random orientation used in data analysis. On the contrary, differences in the hydrostatic conditions are not observed to be significant (Ohtani et al., 2013), unless differential stress results in the development of preferential orientation in the sample (Antonangeli et al., 2010).

The discussion of the different NRIXS studies is somewhat less straightforward, because an EOS is used to solve for $V_{\mathrm{P}}$ from the measured Debye velocity and because of the different isotopic enrichment in the samples used (which becomes relevant when estimating the density from the pressure or the volume). We considered the reported $V_{\mathrm{P}}$ without accounting for possible difference due to the use of different EOS or dissimilar data processing. When not directly measured (as in Mao et al. (2001) and in Lin et al. (2005)), the density has been estimated using the same EOS used for data analysis, in order to have values of $V_{\mathrm{P}}$ and $\rho$ internally consistent, but neglecting the possible influence of variations in isotopic enrichment. For studies reporting data at simultaneous high-pressure, high-temperature conditions (Lin et al., 2005), we only considered $300 \mathrm{~K}$ data. Figure 4 shows $V_{\mathrm{P}}$ as a function of density obtained by NRIXS studies (Mao et al., 2001; Lin et al., 2005; Murphy et al. 2013; Gleason et al., 2013). More recent data sets (Murphy et al. 2013; Gleason et al., 2013) benefited from improved instrument performance and have been collected with improved counting statistics, as well as enhanced hydrostatic conditions, relative to earlier determinations. Furthermore, density has been 


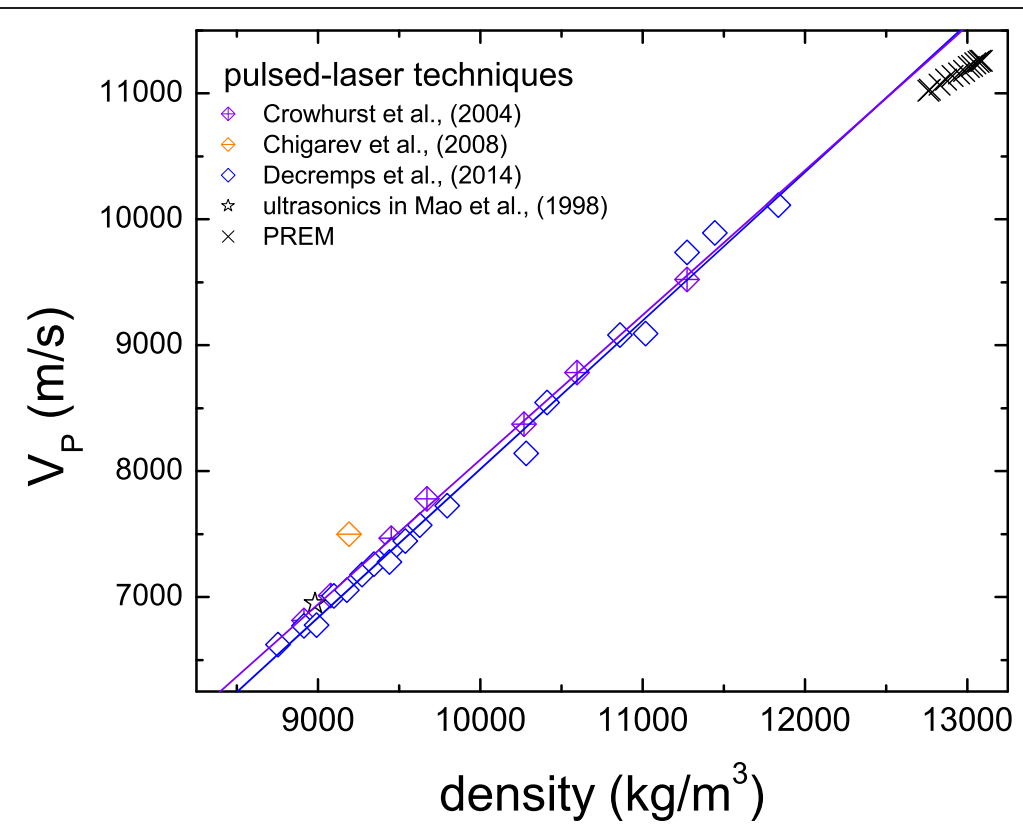

Figure 5 Compressional sound velocity as a function of density as derived from pulsed-laser techniques. Violet crossed diamonds (Crowhurst et al., 2004); orange lined diamond (Chigarev et al., 2008); blue open diamonds (Decremps et al., 2014). Lines are linear fits to the experimental data sets. The star is the ultrasonic determination reported in Mao et al., (1998). PREM is shown as crosses.

directly measured by complementary X-ray diffraction experiments. However, the two data sets, while suggesting a similar slope for the $V_{\mathrm{P}^{-}} \rho$ relation, display the largest difference in the absolute values of the velocities. A direct consequence of this is that one data set extrapolates slightly above PREM (Gleason et al., 2013) while the other extrapolates well below PREM (Murphy et al. 2013) (see Figure 4). A possible cause for this apparent discrepancy might come from the fact that, in contrast to other NRIXS studies, sample isotopic enrichment $(56.95 \mathrm{vs} 55.845 \mathrm{~g} / \mathrm{mol}$ of natural $\mathrm{Fe}$ ) has been explicitly accounted for in density determination in the analysis of Murphy et al. (2013). After rescaling the reported density to those expected for natural Fe (dotted line in Figure 4), we observe a closer agreement, even if the trend from Murphy et al. (2013) is still slightly below the others.

Pulsed laser techniques, even though not systematically used, provide measurements of sound velocities at pressures exceeding the megabar and are complementary to synchrotron-based techniques in many ways. In particular, picosecond acoustics can be seen as the most direct technique for sound velocity measurements in DAC, at least to the extent that, as in conventional pulse-echo ultrasonics, it is based on travel time determination. One of the main current disadvantages of both ISLS and picosecond acoustics is that these laboratory-based techniques are not yet coupled with X-ray diffraction. Accordingly, these measurements are more largely dependent on specific choice of ruby pressure scale and iron EOS. Results obtained by ISLS (Crowhurst et al., 2004) and by picosecond acoustics (Decremps et al., 2014) are reported in Figure 5. Densities have been determined starting with the reported pressures and using the EOS by Mao et al. (1990), which is consistent with the old ruby scale, and then using our reference EOS to reassess the pressure values. We observe that ISLS and picosecond acoustics data sets are in close agreement and linearly extrapolate to values somewhat above PREM.

\section{A reference for $V_{\mathrm{P}}-\rho$ relation for $\mathrm{hcp}-\mathrm{Fe}$ at $300 \mathrm{~K}$}

Combining results obtained by different techniques, each one with its own specific and often very dissimilar advantages and limitations, likely provides the most stringent constraints on the $V_{\mathrm{P}}-\rho$ relation. Application of classic pulse-echo ultrasonic techniques in multi-anvil presses is feasible but challenging, and only a single measurement has been obtained on hcp-Fe, at the relatively low pressure of $16.5 \mathrm{GPa}$ (Mao et al., 1998). As already mentioned, $V_{\mathrm{P}}$ derived from XRD-based techniques suffers from model dependence, but data have been obtained up to pressures close to $300 \mathrm{GPa}$ (Mao et al., 1998; Dubrovinski et al., 2001), supporting a linear dependence of $V_{\mathrm{P}}$ with density. NRIXS measurements may provide the most accurate determination of $V_{\mathrm{S}}$ (Murphy et al., 2013; Gleason et al., 2013), while $V_{\mathrm{P}}$ values are more dependent on the choice of the EOS used in the data analysis. On general grounds, fitting the low-energy portion of the phonon density of state as a parabola, as commonly done, might lead to an underestimation of the Debye velocity (Chumakov, 2014). This, together with the fact 
that measurements are carried on isotopically enriched samples of higher atomic weight than natural iron $\left(56.95 \mathrm{~g} / \mathrm{mol}\right.$ for $95 \%$ isotopically enriched ${ }^{57}$ Fe samples vs. $55.85 \mathrm{~g} / \mathrm{mol}$ for natural iron), might explain, at least qualitatively, why NRIXS measurements yield $V_{\mathrm{P}}$ values generally lower than those measured by other techniques. Furthermore, the most recent, and presumably most accurate, NRIXS measurements (Murphy et al., 2013; Gleason et al., 2013) are in disagreement in terms of $V_{\mathrm{P}}$ determinations (see Figure 4). Accordingly, we will not use NRIXS results to constrain the $V_{\mathrm{P}}-\rho$ relation. IXS in combination with XRD has been largely used to measure the aggregate compressional sound velocity as a function of density. Provided an adequate data collection strategy (energy and momentum resolution, number and relative positions of points to constrain the phonon dispersion curve) and controlled and limited texture of the samples (polycrystalline aggregates, as close as possible to an ideal randomly oriented powder), $V_{\mathrm{P}}$ can be obtained with $1 \%$ to $2 \%$ precision. Amongst the various data sets present in literature, we will not make use of the result by Fiquet et al. (2001), as these pioneering measurements have been superseded by subsequent determinations using improved instrumentation. Similarly, we do not use the results by Mao et al. (2012), because they were obtained on a foil, with relaxed momentum resolution and non-ideal settings of the spectrometer. Furthermore, this last data set is the only one supporting a sub-linear dependence of $V_{\mathrm{P}}$ on $\rho$, in contrast to all other determinations. Both ISLS and picosecond acoustics do not allow for parallel determination of the density (at least with currently available experimental setups), but provide direct determination of surface waves (ISLS) and acoustic echoes (picosecond acoustics) travelling across the sample. With accurate estimation of the sample thickness, picosecond acoustic methods may thus yield the most direct measurements of $V_{\mathrm{P}}$.

Selected measurements of the compressional sound velocity as a function of density are shown in Figure 6. All the data agree within mutual uncertainty and plot along a well-defined linear trend. Linear regression of all the data, according to the relation $V_{\mathrm{P}}=a+b \rho$, yields $a=-4,000 \pm 110 \mathrm{~m} / \mathrm{s}$ and $b=1.206 \pm 0.011 \mathrm{~m}^{4} /(\mathrm{kg} \mathrm{s})$ (solid line in Figure 6). For completeness, the same data and fit are plotted as a function of pressure in Figure 7.

\section{Extrapolation to inner core conditions and high- temperature effects}

The linear fit to the experimentally observed $V_{\mathrm{P}}-\rho$ trend can be extrapolated to inner core densities and compared to PREM (Figure 6). $V_{\mathrm{P}}$ for hcp-Fe at $300 \mathrm{~K}$ is $3 \%$ to $4 \%$ above PREM, with a slightly steeper slope. Inner core temperatures are not well constrained, ranging between 4,000 and 7,000 K (Anzellini et al., 2013; Nomura et al, 2014). Accordingly, temperature effects have to be considered when comparing $300 \mathrm{~K}$ data with seismic observations. Within a quasi-harmonic approximation, $V_{\mathrm{P}}$ is expected to scale linearly with density, irrespective

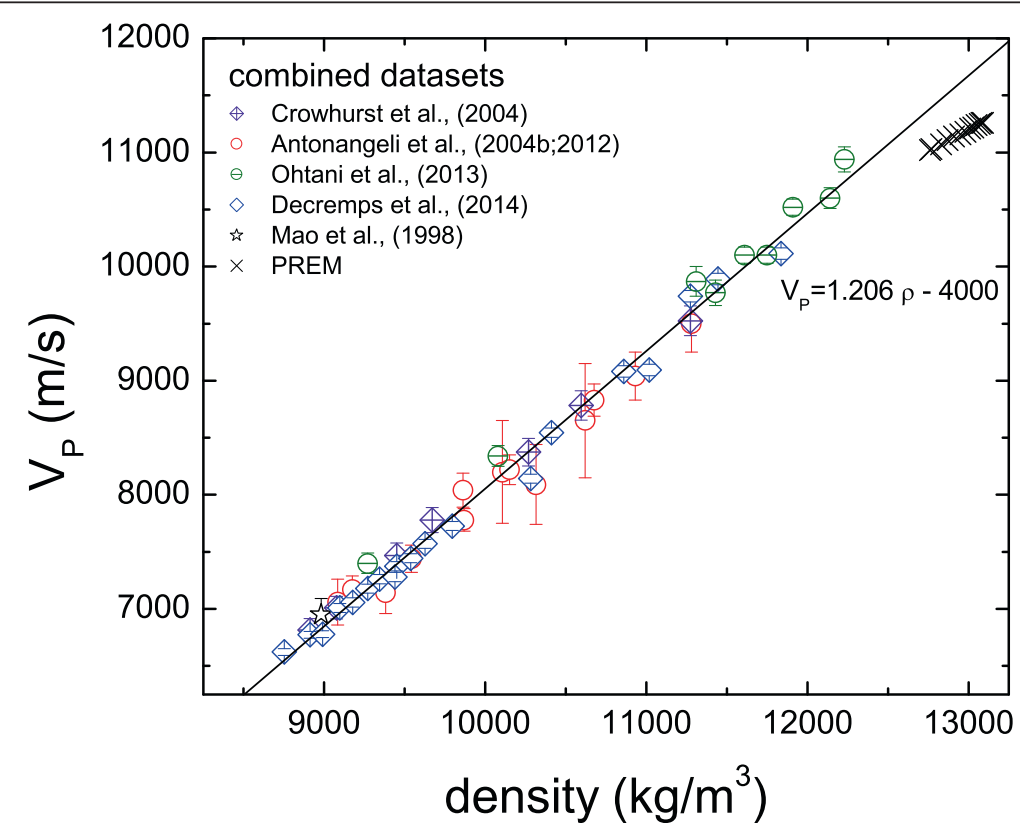

Figure 6 Compressional sound velocity as a function of density. Violet crossed diamonds (Crowhurst et al., 2004); blue open diamonds (Decremps et al., 2014); red open circles (Antonangeli et al., 2004b; 2012); green lined circles (Ohtani et al., 2013). The star is the ultrasonic determination reported in (Mao et al., 1998). PREM is shown as crosses. The solid line is a linear regression across all experimental data $\left(V_{p}=a+b \rho\right.$ where $a=-4,000 \pm 110 \mathrm{~m} / \mathrm{s}$ and $\left.b=1.206 \pm 0.011 \mathrm{~m}^{4} /(\mathrm{kg} \mathrm{s})\right)$. 


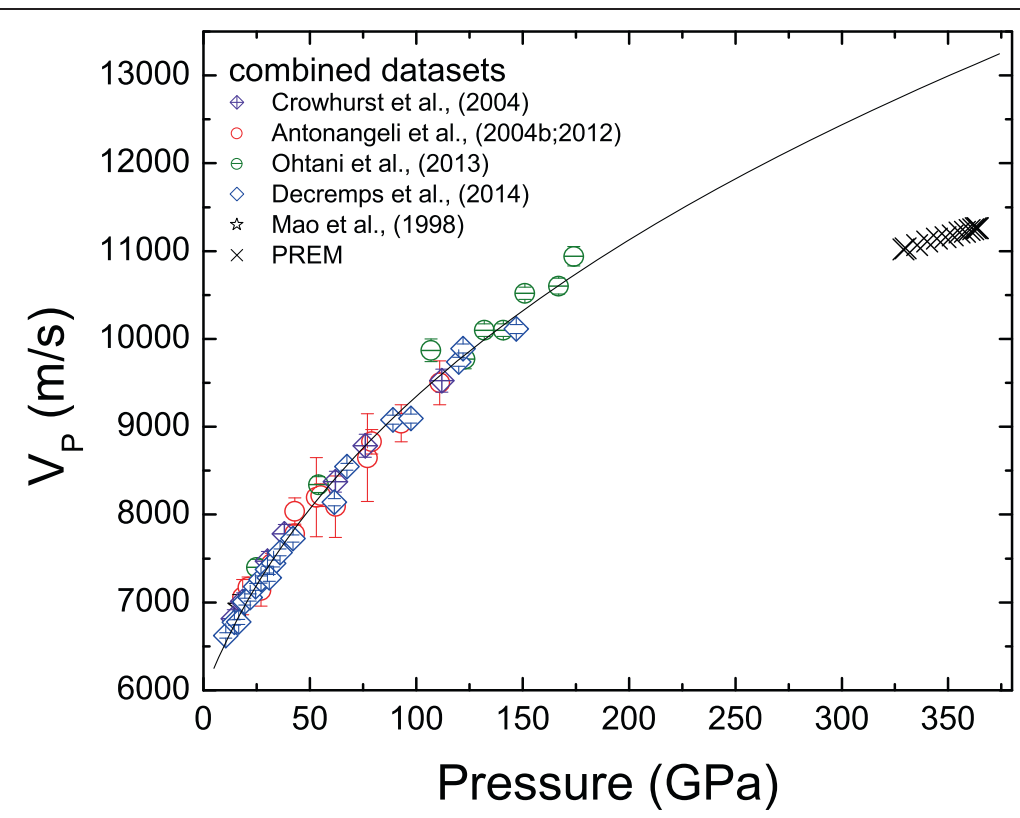

Figure 7 Compressional sound velocity as a function of pressure. Violet crossed diamonds (Crowhurst et al., 2004); blue open diamonds (Decremps et al., 2014); red open circles (Antonangeli et al., 2004b; 2012); green lined circles (Ohtani et al., 2013). The star is the ultrasonic determination reported in Mao et al., (1998). PREM is shown as crosses. The solid line is the extrapolation to higher pressure after the Birch's fit in Figure 6.

of the specific $P-T$ conditions. This relation is often referred to as "Birch's law". Limits in the validity of the Birch's law have been discussed in literature, with specific focus on the case of iron, but without reaching a consensus (Fiquet et al., 2001; Lin et al., 2005; Antonangeli et al.,
2008; 2012; Mao et al., 2012; Ohtani et al., 2013). In all cases, however, investigated temperature ranges were quite limited (1,000 to 1,700 K). Much higher temperatures are instead achievable by shock compression. Ironically, shock wave measurements along the Hugoniot

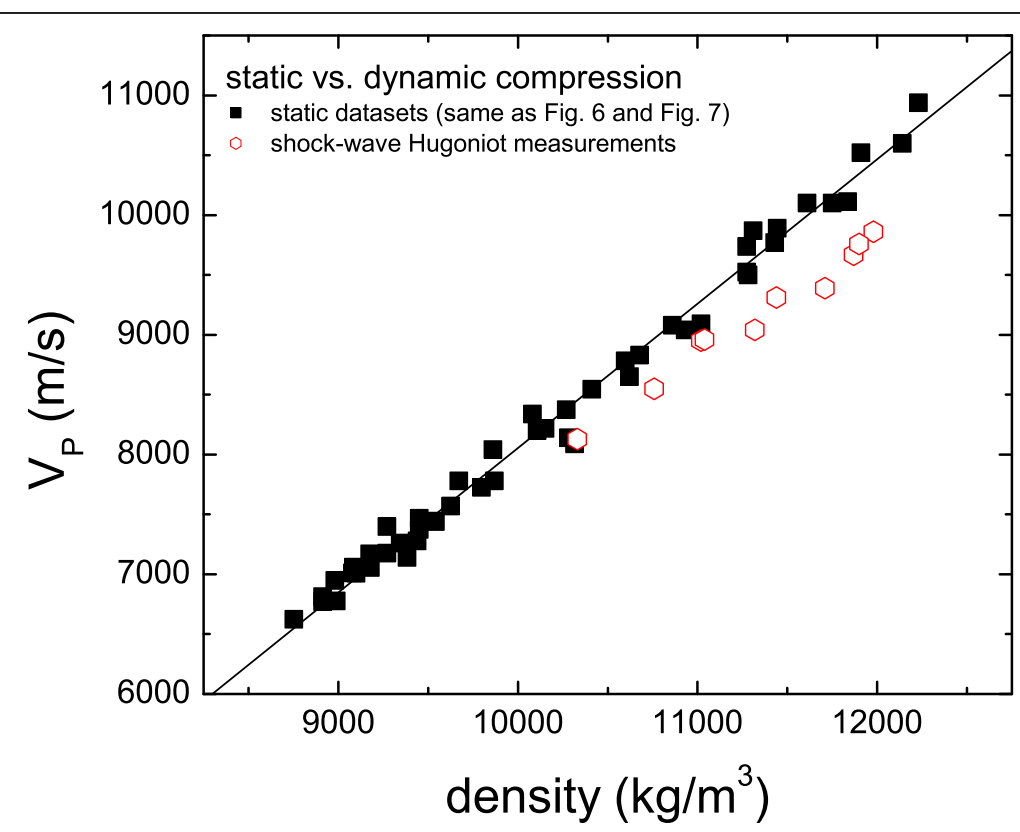

Figure 8 Compressional sound velocity as a function of density. Black squares are selected data sets (same as Figures 6 and 7) obtained under static compression at $300 \mathrm{~K}$ (Mao et al., 1998; Crowhurst et al., 2004; Antonangeli et al., 2004b; 2012; Ohtani et al., 2013; Decremps et al., 2014). The solid line is a linear regression across the static data. Red open hexagons are shock wave Hugoniot measurements not reduced to $300 \mathrm{~K}$ (Brown and McQueen, 1986). 
suffer from the opposite problem: temperatures at elevated pressures are much higher than the geotherm and the Hugoniot curve crosses the iron melting line at approximately 225 GPa (Nguyen and Holmes, 2004). Accordingly, $V_{\mathrm{P}}$ data obtained in shock wave experiments on solid hcp-Fe are limited to pressures below approximately $205 \mathrm{GPa}$ (Brown and McQueen, 1986). The comparison between selected $V_{\mathrm{P}}$ measurements at $300 \mathrm{~K}$ under static compression and shock wave determination at high temperature, along the Hugoniot curve, is reported in Figure 8. Shock data yield systematically lower $V_{\mathrm{P}}$ than static data, with a difference that seems to increase, even if slightly, with increasing density. The most reasonable explanation for this difference is the anharmonic effects at high temperature, which are predicted to decrease velocities even at constant density (albeit a larger reduction is anticipated for $V_{\mathrm{S}}$ than for $V_{\mathrm{P}}$ ) (e.g. Laio et al., 2000; Vočadlo et al., 2009; Sha and Cohen, 2010). Anharmonic effects are expected to increase with increasing temperature, but to decrease with increasing compression, and a quantitative assessment of eventual temperature-induced softening of $V_{\mathrm{P}}$ calls for further experimental work at simultaneous high static pressure and high temperature. In any case, the observed systematic difference between data sets obtained at ambient temperature and shock measurements argues against the suitability of fits combining results of ambient temperature static compression experiments with results by shock wave at high temperature, as proposed by Fiquet et al. (2001) or Badro et al. (2007).

\section{Conclusions}

In this paper, we reviewed the experimental techniques and methodologies used to measure sound velocities in metals at megabar pressures, with specific focus on the compressional sound velocity of hcp-Fe. Each technique presents advantages and drawbacks. Being aware of these is of the greatest importance when assessing the robustness of each measurement and, all the more so, when comparing different data sets. A critical review of literature results, coherently analyzed using consistent metrology (pressure scale, equation of state of hcp-Fe), allows us to propose a reference for the $V_{\mathrm{P}}-\rho$ relationship in hcp-Fe at $300 \mathrm{~K}$ that linearly extrapolates $3 \%$ to $4 \%$ above PREM (Figure 6). This provides the principal base line upon which to add complexity, including anharmonic effects at high temperature, pre-melting effects, effects of nickel and/or light element alloying, required for accurate reconciliation with seismic models. In particular, our results argue for combined effects of high temperature and inner core impurities (such as nickel and light elements) that slightly reduce $V_{\mathrm{P}}$ of pure hcp-Fe at constant density. This can be potentially satisfied by assuming that both alloying and high temperature only very moderately lower $V_{\mathrm{P}}$ which, however, is not supported by recent results (Badro et al., 2007; Antonangeli et al., 2010; Sha and Cohen 2010; Mao et al. 2012; Martorell et al., 2013). Alternatively, and most likely, effects of impurities (combined effect of nickel and light elements) and high temperature counterbalance, the first increasing velocity at constant density (e.g. Antonangeli et al. 2010) and the second decreasing velocity at constant density (e.g. Sha and Cohen 2010), leading to slightly reducing overall sum.

\section{Competing interests}

The authors declare that they have no competing interests.

\section{Authors' contribution}

DA and EO planned the research and reviewed literature data. DA analyzed the data and prepared the figures. Both authors discussed the results and contributed in writing the paper. Both authors read and approved the final manuscript.

\section{Acknowledgements}

DA acknowledges the financial support from the Center of Excellence (COE) programme of Tohoku University for his visit to Japan in February 2014 that provided the ground for the discussions that led to the ideas presented in this paper. The authors wish to thank FJ Ryerson for proofreading the manuscript. This work has been supported by the French National Research Agency (ANR) grant no. 2010-JCJC-604-01 (DA), the JSPS KAKENHI (Grant-in-Aid for Specially Promoted Research) Grant Number 22000002, and the Ministry of Education and Science of Russian Federation, Project 14.B25.31.0032 (EO).

\section{Author details}

${ }^{1}$ Institut de Minéralogie, de Physique des Matériaux, et de Cosmochimie (IMPMC), UMR CNRS 7590, Sorbonne Universités - UPMC Univ. Paris 6, Muséum National d'Histoire Naturelle, IRD unité 206, 75252 Paris, France. ${ }^{2}$ Department of Earth and Planetary Materials Science, Graduate School of Science, Tohoku University, Sendai, Miyagi 980-8570, Japan.

Received: 18 September 2014 Accepted: 17 February 2015

Published online: 13 March 2015

\section{References}

Antonangeli D, Krish M, Fiquet G, Farber DL, Aracne CM, Badro J, Occelli F, Requardt H (2004a) Elasticity of cobalt at high pressure studied by inelastic x-ray scattering. Phys Rev Lett 93:215505

Antonangeli D, Occelli F, Requardt H, Badro J, Fiquet G, Krisch M (2004b) Elastic anisotropy in textured hcp-iron to $112 \mathrm{GPa}$ from sound velocity wave propagation measurements. Earth Planet Sci Lett 225:243-251

Antonangeli D, Krisch M, Farber DL, Ruddle DG, Fiquet F (2008) Elasticity of hexagonal-closed-packed cobalt at high pressure and temperature: a quasiharmonic case. Phys Rev Lett 100:085501

Antonangeli D, Siebert J, Badro J, Farber DL, Fiquet G, Morard G, Ryerson FJ (2010) Composition of the Earth's inner core from high-pressure sound velocity measurements in Fe-Ni-Si alloys. Earth Planet Sci Lett 295:292-296

Antonangeli D, Komabayashi T, Occelli F, Borissenko E, Walters AC, Fiquet G, Fei Y (2012) Simultaneous sound velocity and density measurements of hcp iron up to $93 \mathrm{GPa}$ and $1100 \mathrm{~K}$ : an experimental test of the Birch's law at high temperature. Earth Planet Sci Lett 331:210-214

Anzellini S, Dewaele A, Mezouar M, Loubeyre P, Morard G (2013) Melting of iron at Earth's inner core boundary based on fast x-ray diffraction. Science 340:464-466

Badro J, Fiquet G, Guyot F, Gregoryanz E, Occelli F, Antonangeli D, d'Astuto M (2007) Effect of light elements on the sound velocities in solid iron: implications for the composition of the Earth's core. Earth Planet Sci Lett 254:233-238

Birch F (1952) Elasticity and constitution of the Earth's interior. J Geophys Res 57:227-286

Bosak A, Krisch M, Fischer I, Huotari S, Monaco G (2007) Inelastic x-ray scattering from polycrystalline materials at low momentum transfer. Phys Rev B 75:064106 
Brown JM, McQueen RG (1986) Phase transition, Grüneisen parameter, and elasticity for shocked iron between $77 \mathrm{GPa}$ and $400 \mathrm{GPa}$. J Geophys Res 91:7485-7494

Chigarev N, Zinin P, Ming LC, Amulele G, Bulou A, Gusev V (2008) Laser generation and detection of longitudinal and shear acoustic waves in a diamond anvil cell. Appl Phys Lett 93:181905

Chumakov A (2014) Possible problems in the determination of the mean sound velocity from the density of vibrational states, Paper presented at RIKENEN Spring-8 Center Workshop on Elastic Properties of Iron in Extreme Conditions via X-Ray Scattering, Kobe, Japan 25-27 February 2014

Crowhurst JC, Goncharov AF, Zaug JM (2004) Impulsive stimulated light scattering from opaque materials at high pressure. J Phys Condens Matter 16:S1137-S1142

Crowhurst JC, Antonangeli D, Brown JM, Goncharov AF, Farber DL, Aracne CM (2006) Determination of the high pressure elasticity of cobalt from measured interfacial acoustic wave velocities. Appl Phys Lett 89:111920

Decremps F, Belliard L, Perrin B, Gauthier M (2008) Sound velocity and absorption measurements under high pressure using picosecond ultrasonics in a diamond anvil cell: application to the stability study of AIPdMn. Phys Rev Lett 100:035502

Decremps F, Antonangeli D, Gauthier M, Ayrinhac S, Morand M, Le Marchand G, Bergame F, Philippe J (2014) Sound velocity measurements of iron up to 152 GPa by picosecond acoustics in diamond anvil cell. Geophys Res Lett 41:1459

Dewaele A, Loubeyre P, Mezouar M (2004) Equations of state of six metals above 94 GPa. Phys Rev B 70:094112

Dewaele A, Loubeyre P, Occelli F, Mezouar M, Dorogokupets PI, Torrent M (2006) Quasihydrostatic equation of state of iron above 2 Mbar. Phys Rev Lett 97:215504

Dubrovinski LS, Dubrovinskaia NA, Le Bihan T (2001) Aggregate sound velocities and acoustic Grüneisen parameter of iron up to $300 \mathrm{GPa}$ and $1200 \mathrm{~K}$. Proc Natl Acad Sci U S A 98:9484-9489

Dziewonski AM, Anderson DL (1981) Preliminary reference Earth model. Phys Earth Planet Inter 25:297-356

Fei Y, Ricolleau A, Frank M, Mibe K, Shen G, Prakapenka V (2007) Toward an internally consistent pressure scale. Proc Natl Acad Sci U S A 104:9182-9186

Fiquet G, Badro J, Guyot F, Requardt H, Krisch M (2001) Sound velocities in iron to 110 Gigapascals. Science 291:468-471

Fiquet G, Badro J, Gregoryanz E, Fei Y, Occelli F (2009) Sound velocity in iron carbide $\left(\mathrm{Fe}_{3} \mathrm{C}\right)$ at high pressure: implications for the carbon content in the Earth's inner core. Phys Earth Planet Inter 172:125-129

Gao L, Chen B, Wang J, Alp EE, Zhao J, Lerche M, Sturhahn W, Scott HP, Huang F, Ding Y, Sinogeikin SV, Lundstrom CC, Bass JD, Lie J (2008) Pressure-induced magnetic transition and sound velocities of $\mathrm{Fe}_{3} \mathrm{C}$ : implications for carbon in the Earth's inner core. Geophys Res Lett 35:L17306

Gleason AE, Mao WL, Zhao JY (2013) Sound velocities for hexagonally closepacked iron compressed hydrostatically to $136 \mathrm{GPa}$ from phonon density of states. Geophys Res Lett 40:2983-2987

Jackson I, Gerald JF, Kokkonen H (2000) High-temperature viscoelastic relaxation in iron and its implications for the shear modulus and attenuation of the Earth's inner core. J Geophys Res 1105:23605-23634

Jacobsen SD, Spetzler H, Reichmann HJ, Smyth JR, Hemley RJ (2004) Shear waves in the diamond-anvil cell reveal pressure-induced instability in (Mg, Fe)O. Proc Natl Acad Sci U S A 101:5867-5871

Kamada S, Ohtani E, Fukui H, Sakai T, Terasaki H, Takahashi S, Shibazaki Y, Tsutsui S, Baron AQR, Hirao N, Ohishi Y (2014) The sound velocity measurements of $\mathrm{Fe}_{3} \mathrm{~S}$. Am Min 99:98-101

Kantor AP, Kantor IY, Kurnusov AV, Kuznetsov AY, Dubrovinskaia NA, Krisch M, Bossak AA, Dmitriev VP, Urusov VS, Dubrovinsky LS (2007) Sound wave velocities of fcc Fe-Ni alloy at high pressure and temperature by mean of inelastic X-ray scattering. Phys Earth Planet Inter 164:83-89

Laio A, Bernard S, Chiarotti GL, Scandolo S, Tosatti E (2000) Physics of iron at Earth's core conditions. Science 287:1027-1030

Lin JF, Struzhkin W, Sturhahn W, Huang E, Zhao J, Hu MY, Alp EE, Mao HK, Boktor N, Hemley RJ (2003) Sound velocity of iron-nickel and iron-silicon alloys at high pressure. Geophys Res Lett 30:2212

Lin JF, Sturhahn W, Zhao J, Shen G, Mao HK, Hemley RJ (2005) Sound velocities of hot dense iron: Birch's law revisited. Science 308:1892-1894

Lin JF, Mao Z, Hyavaş H, Zhao J, Dubrovinsky L (2010) Shear wave anisotropy of textured hcp-Fe in the Earth's inner core. Earth Planet Sci Lett 298:361-366
Mao KH, Wu Y, Chen LC, Shu JF, Jephcoat AP (1990) Static compression of iron to $300 \mathrm{GPa}$ and $\mathrm{Fe}_{0.8} \mathrm{Ni}_{0.2}$ alloy to $260 \mathrm{GPa}$ : implications for composition of the core. J Geophys Res 95:21737-21742

Mao HK, Shu J, Shen G, Hemley RJ, Li B, Singh AK (1998) Elasticity and rheology of iron above $220 \mathrm{GPa}$ and the nature of the Earth's inner core. Nature 396 : 741-743; correction (1999). Nature 399:80

Mao HK, Xu J, Struzhkin W, Shu J, Hemley RJ, Sturhahn W, Hu MY, Alp EE, Vočadlo L, Alfè D, Price GD, Gillian MJ, Schwoerer-Böhning M, Häusermann D, Eng P, Shen G, Giefers H, Lübbers R, Wortmann G (2001) Phonon density of states of iron up to $153 \mathrm{GPa}$. Science 292:914-916

Mao Z, Lin JF, Liu J, Alatas A, Gao L, Zhao J, Mao HK (2012) Sound velocities of Fe and Fe-Si alloy in the Earth's core. Proc Natl Acad Sci U S A 109:10239-10244

Martorell B, Vočadlo L, Brodholt J, Wood IG (2013) Strong premelting effect in the elastic properties of hcp-Fe under inner-core conditions. Science 342:466-468

Matsui M, Ito E, Katsura T, Yamazaki D, Yoshino T, Yokoyama A, Funakoshi K (2009) The temperature-pressure-volume equation of state of platinum. J Appl Phys 105:013505

Merkel S, Miyajima N, Antonangeli D, Fiquet G, Yagi T (2006) Lattice preferred orientation and stress in polycrystalline hcp-Co plastically deformed under high pressure. J Appl Phys 100:023510

Merkel S, Tomé C, Wenk H-R (2009) Modeling analysis of the influence of plasticity on high pressure deformation of hcp-Co. Phys Rev B 79:064110

Murakami M, Ohishi Y, Hirao N, Hirose K (2012) A perovskitic lower mantle inferred from high-pressure high-temperature sound velocity data. Nature 485:90-94

Murphy CA, Jackson JM, Sturhahn W (2013) Experimental constraints on the thermodynamics and sound velocities of hcp-Fe to core pressures. J Geophys Res Solid Earth 118:1-18

Nguyen JH, Holmes NC (2004) Melting of iron at the physical conditions of the Earth's core. Nature 427:339-342

Nomura R, Hirose K, Uesugi K, Ohishi Y, Tsuchiyama A, Miyake A, Ueno Y (2014) Low core-mantle boundary temperature inferred from the solidus of pyrolite. Science 343:522-525

Ohtani E, Shibazaki Y, Sakai T, Mibe K, Fukui H, Kamada S, Sakamaki T, Seto Y, Tsutsui S, Baron AQR (2013) Sound velocity of hexagonal close-packed iron up to core pressures. Gephys Res Lett 40:5089-5094

Sakai T, Ohtani E, Hirao N, Ohishi Y (2011) Equation of state of the NaCl-B2 phase up to 304 GPa. J Appl Phys 109:084912

Sakai T, Takahashi S, Nishitani N, Mashini I, Ohtani E, Hirao N (2014) Equation of state of pure iron and $\mathrm{Fe}_{0.9} \mathrm{Ni}_{0.1}$ alloy up to $3 \mathrm{Mbar}$. Phys Earth Planet Inter 228:114-126

Sha X, Cohen RE (2010) First-principles thermal equation of state and thermoelasticity of hcp Fe at high pressures. Phys Rev B 81:094105

Shibazaki Y, Ohtani E, Fukui H, Sakai T, Kamada S, Ishikawa D, Tsutsui S, Baron AQR, Nishitani N, Hirao N, Takemura K (2012) Sound velocity measurements in dhcp-FeH up to $70 \mathrm{GPa}$ with inelastic $x$-ray scattering: implications for the composition of the Earth's core. Earth Planet Sci Lett 313-314:79-85

Sturhahn W, Jackson JM (2007) Geophysical applications of nuclear resonant spectroscopy. Geol. Soc. Am., Special Paper 421: 157-174.

Tateno S, Hirose K, Ohishi Y, Tatsumi Y (2010) The structure of iron in Earth's inner core. Science 330:359-361

Vočadlo $L$ (2007) Ab initio calculations of the elasticity of iron and iron alloys at inner core conditions: evidence of partially molten core? Earth Planet Sci Lett 288:534-538

Vočadlo L, Dobson DP, Wood IG (2009) Ab initio calculations of the elasticity of hcp-Fe as a function of temperature at inner-core pressure. Earth Planet Sc Lett 288:534-538 